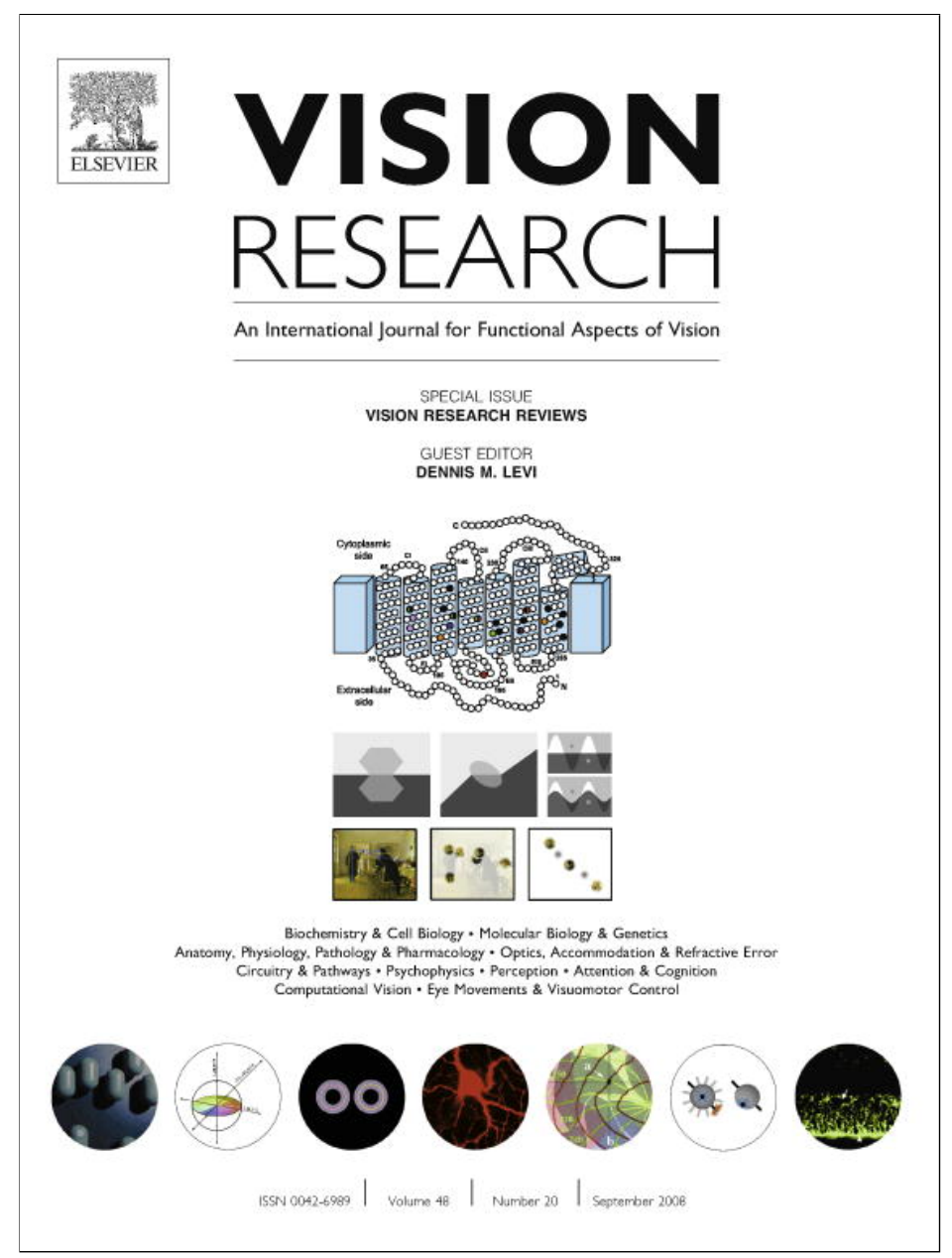

This article appeared in a journal published by Elsevier. The attached copy is furnished to the author for internal non-commercial research and education use, including for instruction at the authors institution and sharing with colleagues.

Other uses, including reproduction and distribution, or selling or licensing copies, or posting to personal, institutional or third party websites are prohibited.

In most cases authors are permitted to post their version of the article (e.g. in Word or Tex form) to their personal website or institutional repository. Authors requiring further information regarding Elsevier's archiving and manuscript policies are encouraged to visit:

http://www.elsevier.com/copyright 


\title{
The role of areas MT and MST in coding of visual motion underlying the execution of smooth pursuit
}

\author{
Uwe J. Ilg* \\ Department of Cognitive Neurology, Hertie-Institute of Clinical Brain Research, University of Tuebingen, Otfried-Mueller-Street 27, D-72076 Tuebingen, Germany
}

\section{A R T I C L E I N F O}

\section{Article history:}

Received 25 January 2008

Received in revised form 8 April 2008

\section{Keywords:}

Posterior parietal cortex

Rhesus monkey

Eye movements

Motion processing

\begin{abstract}
A B S T R A C T
What is the main purpose of visual motion processing? One very important aspect of motion processing is definitively the generation of smooth pursuit eye movements. These eye movements avoid motion blur of moving objects which would obstruct the analysis of the objects' visual details. However, these eye movements can only be executed if there is a moving target. So there is a very close and inseparable relationship between smooth pursuit and motion processing. The hub for visual motion processing is situated in the middle temporal (MT) and medial superior temporal (MST) area. Despite the undoubted importance of these areas for the generation of smooth pursuit or goal-directed behavior in general, it is important to keep in mind that motion processing in addition serves perceptual purposes such as object recognition, structure-from-motion detection, scene segmentation, self-motion estimation and depth perception. This review focuses at the beginning on pursuit-related activity recorded from MT and MST, subsequently extends the view to goal-directed hand movements, and finally addresses the possible contributions of these areas to motion perception.
\end{abstract}

(c) 2008 Elsevier Ltd. All rights reserved.

\section{Basic features of neuronal responses recorded from MT and MST}

The middle temporal area (MT) in the posterior bank of the superior temporal sulcus (STS) was first described more than 30 years ago (Allman \& Kaas, 1971; Dubner \& Zeki, 1971). MT can be characterized by two key features: first, this area is anatomically defined by the intensive staining of myelin. Second, almost all neurons respond to visual motion in a direction-selective manner. If a visual stimulus, usually a group of coherent moving dots within a stationary aperture adjusted to the location of the receptive field, moves in the preferred direction, the firing rate of the neuron increases above spontaneous activity. In contrast, if the stimulus moves in the opposite direction, the firing rate is decreased below spontaneous activity (see Fig. 1A). If more directions of motion are tested, the directional tuning of the neuron can be determined (see Fig. 1B). Frequently, this directional tuning is described by a circular von Mises function. In addition to the sensitivity to direction, MT neurons are also sensitive to the speed of the visual stimulation (see Fig. 1C). Interestingly, the inhibition caused by stimulus movement in the non-preferred direction is constant and not speed-dependent.

Some years later, the adjacent medial superior temporal area (MST) in the STS was described (Desimone \& Ungerleider, 1986;

\footnotetext{
* Fax: +497071295724

E-mail address: uwe.ilg@uni-tuebingen.de
}

Tanaka et al., 1986). MST receives direct input from MT (Maunsell \& van Essen, 1983; Ungerleider \& Desimone, 1986) and is composed of two different parts: the lateral aspect extending mainly to the fundus of the STS (MSTl), and the dorsal part localized in the anterior bank of the STS (MSTd) (Mikami, Newsome, \& Wurtz, 1986). MSTl contains neurons with rather small receptive fields and is important for the execution of smooth pursuit eye movements. A large part of this review addresses the role of MSTl. On the other hand, MSTd contains neurons with much larger receptive fields and is most likely important for the analysis of optic flow elicited by self-motion through a highly structured environment (Bradley, Maxwell, Andersen, Banks, \& Shenoy, 1996; Duffy \& Wurtz, 1991).

An important question asks whether the motion sensitivity observed in area MT is established by local circuits in this area or whether it is determined earlier in processing. A peculiarity of primates is that directionally selective ganglion cells are absent in the primate retina. Directionally selective neurons are recorded from $\mathrm{V} 1$, the first stage of cortical processing of visual information (Hawken, Parker, \& Lund, 1988; Hubel \& Wiesel, 1968). There is experimental evidence that exclusively these directionally selective neurons in V1 provide the direct input to MT (Movshon \& Newsome, 1996). In addition, there is indirect input from V1 to MT via V2 (Ponce, Lomber, \& Born, 2008). This indirect route seems to be especially important for the selectivity of MT neurons for the processing of disparity, i.e. the differences in left and right eye images. Finally, there are also descriptions of a direct input to 
MT from the LGN (Nassi, Lyon, \& Callaway, 2006; Sincich, Park, Wohlgemuth, \& Horton, 2004) whose function is not entirely clear at present.

\section{Details of pursuit-related activity recorded from MT and MST neurons}

In order to demarcate visual and extra-retinal signals contributing to the observed pursuit-related activity, we decided to use real and imaginary targets. The real target consists of an hour-glass-like figure; its height was adjusted to $20^{\circ}$. We trained our monkeys to direct their gaze towards the intersection of the two diagonals. If the target abruptly changed its position, the monkeys performed saccades. If the target moved at constant velocity, the monkeys performed smooth pursuit. In the next step, we created the imaginary target by removing the central part of the real figure $\left(12^{\circ}\right)$, in particular the intersection. We adjusted the size of the blanked area to the typical size of receptive fields of visual tracking (VT) neurons recorded from MSTl. In order to direct their gaze towards this imaginary target, the monkeys had to reconstruct the invisible intersection of diagonals using the peripheral visual stimulation. Whenever the monkeys fixated the line endings, the ongoing trial was aborted because of violation of the eye control window. At the outset, we carefully examined the oculo-motor repertoire of our monkeys (Ilg \& Thier, 1999).

In short, we observed that saccade latencies were slightly increased for changes in the position of imaginary targets compared to real targets. With respect to smooth pursuit, pursuit onset latency proved to be similar for real and imaginary targets. The initial acceleration was markedly reduced in the case of the imaginary target. When we analyzed the steady-state pursuit gain, we found comparable gain values during pursuit of the real and the imaginary hour-glass and imaginary triangle. This imaginary triangle consists of the upper half of the imaginary hour-glass and therefore did not provide foveal enclosure of the pursuit target. The gain markedly dropped if the monkey was asked to pursue a single dot para-foveally. We concluded from these data that precisely adjusted pursuit eye movements were achieved whenever a figure, independent of whether it was a real or imaginary figure, was tracked.

Subsequently, we recorded pursuit-related activity during tracking of the real and imaginary target from MT and MSTl (Ilg \& Thier, 2003). We applied the following statistical criteria to evaluate the pursuit-related responses. First, pursuit-related activity during smooth pursuit in the preferred direction of the recorded neuron had to be significantly larger as during pursuit in the opposite, non-preferred direction. 82 neurons from MT and 356 neurons

Fig. 1. Responses of a typical MT neuron to visual motion (unit 205.1, recordings performed by Inés Trigo-Damas). (A) The response to rightward and leftward motion of a random dot pattern (velocity $25^{\circ} / \mathrm{s}$, size $15^{\circ}, 1 \mathrm{~s}$ each, located on the receptive field centered $18^{\circ}$ left on the horizontal meridian). The activity of every single trial is shown as a raster display; the summed activity of all trials is displayed as spike density function (width $20 \mathrm{~ms}$ ). The grey horizontal lines denote the mean activity caused by rightward and leftward movement, respectively. The latency of the response was $60 \mathrm{~ms}$. (B) The resulting directional tuning of this neuron in a polar plot. Every blue dot represents the mean activity caused by movement of the stimulus in this direction. The red profile shows the fitted von Mises function $\mathrm{f}_{(\mathrm{dir})}=\mathrm{A} /\left(2 * \Pi * \mathrm{I}_{0(\mathrm{k})}\right) * e^{(\mathrm{k} *(\cos (\mathrm{dir}-\mathrm{T})))}$. The grey circle indicates the spontaneous activity. The preferred direction of this neuron was to the right. (C) The tuning to speed of this neuron; red represents rightward (=preferred) direction, blue leftward movement. The excitation elicited by a stimulus moving in the preferred direction can be expressed as $\mathrm{f}_{(\mathrm{vel})}=\mathrm{A} * \operatorname{vel}^{\mathrm{B}} * \mathrm{e}^{-(\mathrm{vel} * \mathrm{C})}$. Note that there was a clear modulation of activity in the preferred direction (Kruskal Wallis test of the activities for the different velocities results in $p=0.0021)$, but no modulation was present in the nonpreferred direction (Kruskal Wallis test $p=0.0985$ ). The preferred velocity of this neuron was $52^{\circ} / \mathrm{s}$. (For interpretation of the references to colour in this figure legend, the reader is referred to the web version of this article.) from MSTl meet this criterion. Second, we tested whether the activity during pursuit of the real target in the preferred direction was significantly different from the activity observed during pursuit of the imaginary target. All neurons recorded from MT showed a significant difference here, emphasizing the visual nature of this response in MT. In contrast, the activity of 178 neurons from MSTl showed no significant difference during pursuit of the real and

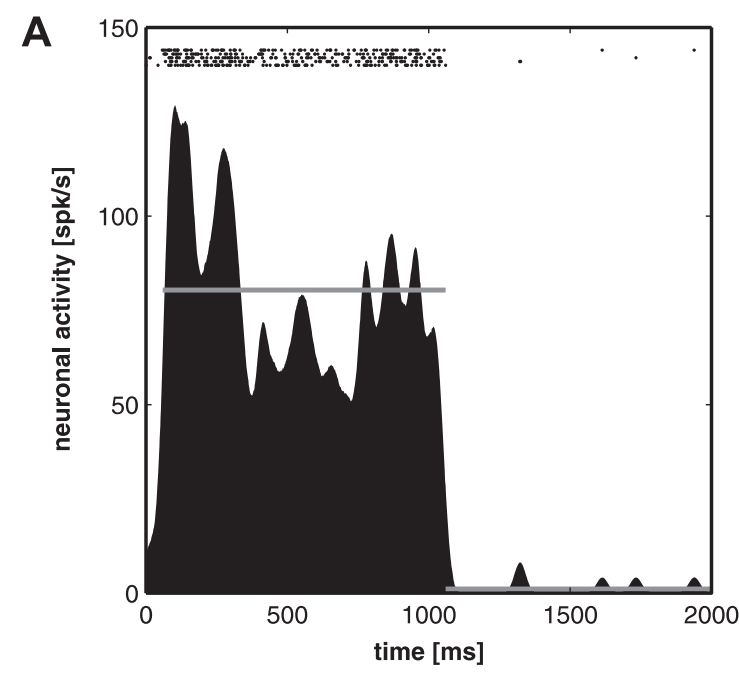

B

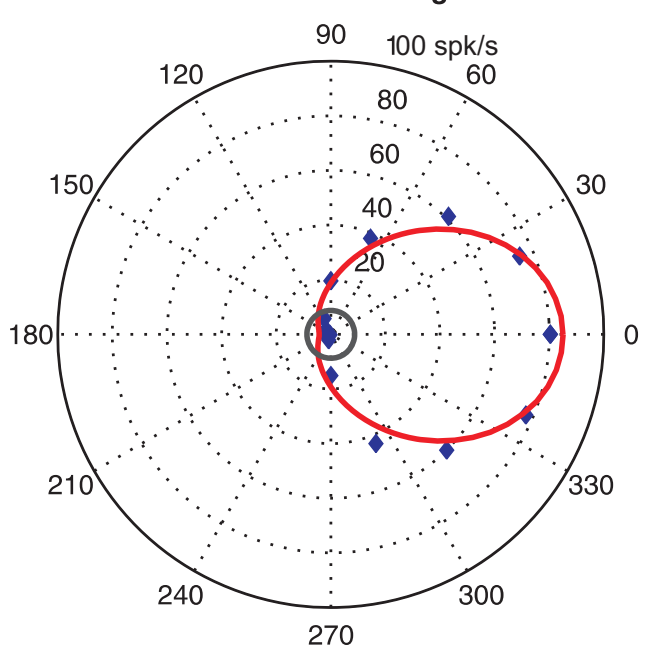

C

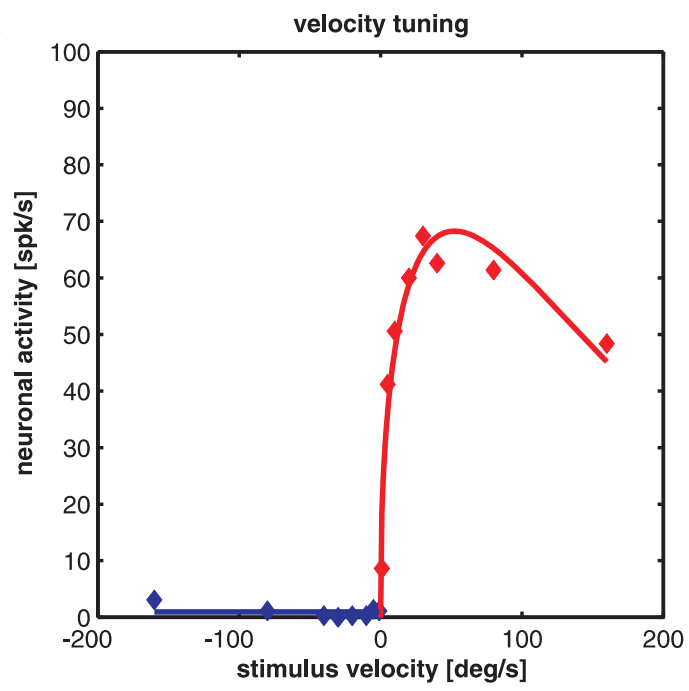


imaginary target. It should be noted that this similarity of responses could in theory also be due to peripheral stimulation by the imaginary target if the neurons would exhibit very large receptive fields. In order to clarify this point, we used a passive visual stimulation. We moved the imaginary target back and forth while the monkey fixated a stationary target. Eighty-five out of the 178 neurons did not respond to this manipulation: in other words, the response of these neurons observed during pursuit of the imaginary target could not be explained by peripheral visual stimulation. In accordance with earlier studies (Kawano, Sasaki, \& Yamashita, 1984), we termed these neurons visual tracking (VT) neurons to emphasize their visual and eye-movement-related responses. The remaining 93 neurons responded only to retinal image motion, they share this property with neurons recorded from MT. The presence of extra-retinal signals in the activity recorded from MST and its absence in the activity of MT neurons was previously documented by experiments performed by Robert Wurtz and his colleagues (Newsome, Wurtz, \& Komatsu, 1988). Instead of spatially removing visual stimulation, they removed the pursuit target briefly in time to test whether the pursuit-related activity contains extra-retinal signals.

In order to examine possible existing head-movement-related signals, it is important to suppress the vestibulo-ocular reflex (VOR). If the VOR would not be suppressed, eye movements in the opposite direction to the head movements occur and possibly cancel the response to the head movement. It is impossible to suppress the VOR voluntarily. However, if a target moves identical to the head, the VOR can be suppressed by fixation of this target. In 13 VT neurons recorded from MSTl, we were able to analyze the responses to head movements while the monkeys were sinusoidally rotated around the yaw or inter-aural axis depending on the preferred direction of the neuron. The monkeys fixated a small target which moved together with the monkey sitting on a turntable. The neuronal activity showed a clear modulation during this manipulation as reported earlier (Thier \& Erickson, 1992). The activity of VT neurons recorded from MSTl is therefore affected by three different parameters: retinal image motion, ongoing eye and head movements. It is important to note that the majority of VT neurons showed identical preferred directions for these three inputs. In contrast, the activity of MT neurons did not show these extra-retinal response qualities; these neurons responded exclusively to retinal image motion. However, for reasons of completeness, the effect of directing attention to the visual stimulation on neuronal responses in MT should briefly be addressed. The response of MT neurons to visual motion is increased if the monkey is attending to the stimulus (Treue \& Maunsell, 1996). Obviously, this attentional effect is another extra-retinal response property which is present in MT as well as in MST neurons.

Since VT neurons in MST receive inputs related to retinal image motion, eye and head movements, their activity is able to encode target trajectory in world-centered coordinates (see Fig. 3). It should be noted here again that only VT neurons have these response properties; many other neurons responded exclusively to visual motion, for instance. In a recently developed model based on the assumption of iso-directionality of the visual, eye and head movement-related responses and similar tuning functions, very realistic tracking parameters of the model were obtained (Dicke \& Thier, 1999). These parameters included steady-state pursuit velocity and initial acceleration as well as eye velocity deficits following unilateral lesions. In contrast to the approach stemming from the idea that MST neurons represent target motion in space, smooth pursuit eye movements could be conceptualized as a negative feedback system operating on retinal image motion of the pursuit target. The main advantage of this concept is its simplicity. The output of these retinal models is very close to the actual parameters of smooth pursuit. However, there are three arguments why it is not very likely that the brain generates smooth pursuit within such a retina-based feedback system: First, seminal experiments performed by Emilio Bizzi and colleagues showed that combined eye and head movements are guided by an internal representation of target movement in space, not within a retinal frame of reference (Lanman, Bizzi, \& Allum, 1978). Second, the presence of eye-movement-related signals as shown above also contradict the assumption of processing within a retinal frame of reference. Third, the perception of our environment during execution of pursuit is clearly not dependent on a retinal frame. Despite self-induced image motion on the retina, we perceive our surrounds stationary as they are in reality. It does not seem very plausible that the brain uses two different reference frames for action and perception.

Finally, the activity of VT neurons could only be casually related to the generation of smooth pursuit if the latency of the neuronal responses was shorter than the eye-movement latency. On average, the latency of the neuronal response to the real and imaginary target was not significantly different (in the order of $120 \mathrm{~ms}$ ), but nevertheless shorter than the eye-movement latency (in the order of $170 \mathrm{~ms}$ ). However, some neurons had longer latencies than the eye movements. As these neurons could not be directly involved in the generation of pursuit, they might be important for signaling eye movements to other parts of the brain that are necessary for the correct processing of self-induced retinal motion.

\section{More experimental evidence for the existence of extra-retinal signals}

The demonstration of eye-movement-related signals in the activity of VT neurons in MSTl is crucial for the argument that these neurons use an external frame of reference. To corroborate the existence of the eye-movement-related signals, we asked whether there were other possibilities to prove the existence of these signals in the activity of VT neurons. If the appearance of a moving target is predictable, human subjects are able to perform anticipatory pursuit (Barnes \& Asselman, 1991; Kowler \& Steinman, 1979). Very recently, we have shown that the ability to perform anticipatory pursuit is similar in human and rhesus monkeys (Freyberg \& Ilg, 2007). Since anticipatory pursuit initiation depends exclusively on prediction and not on actual visual motion signals, this situation can also be used to document extra-retinal responses. To examine anticipatory pursuit, we applied a paradigm in which the target moved periodically (period of $3 \mathrm{~s}$ ) from $9^{\circ}$ left to $9^{\circ}$ right at $12 \% \mathrm{~s}$ and vice versa (Ilg, 2003). After the training, in which the target was continuously visible, the test stimulus was shown during which the target was only visible from $4.5^{\circ}$ left to $4.5^{\circ}$ right with the same periodicity. We determined mean eye speed in $100 \mathrm{~ms}$ intervals around the predictable appearance of the target. We contrasted the prediction condition with a control condition in which the monkey could not anticipate the appearance of the target. The eye velocity was significantly higher in the first three time bins of the prediction conditions (see Fig. 2A and B). Only $200 \mathrm{~ms}$ after target appearance, when the visual motion signal of the target was available, the significant differences in eye speed disappeared.

We recorded from 27 neurons in MSTl in which we were able to document extra-retinal responses by display of the imaginary target (VT neurons) and from 19 neurons lacking this response property (nVT neurons). When the population response of VT neurons obtained during the anticipatory eye movements was compared to the response in the control condition, a difference in latency was apparent (see Fig. 2C). The latency was significantly shorter in the prediction condition compared to the control condition. This difference in latency was absent in the population response of the nVT neurons (see Fig. 2D). The reason for the shorter latency in the 

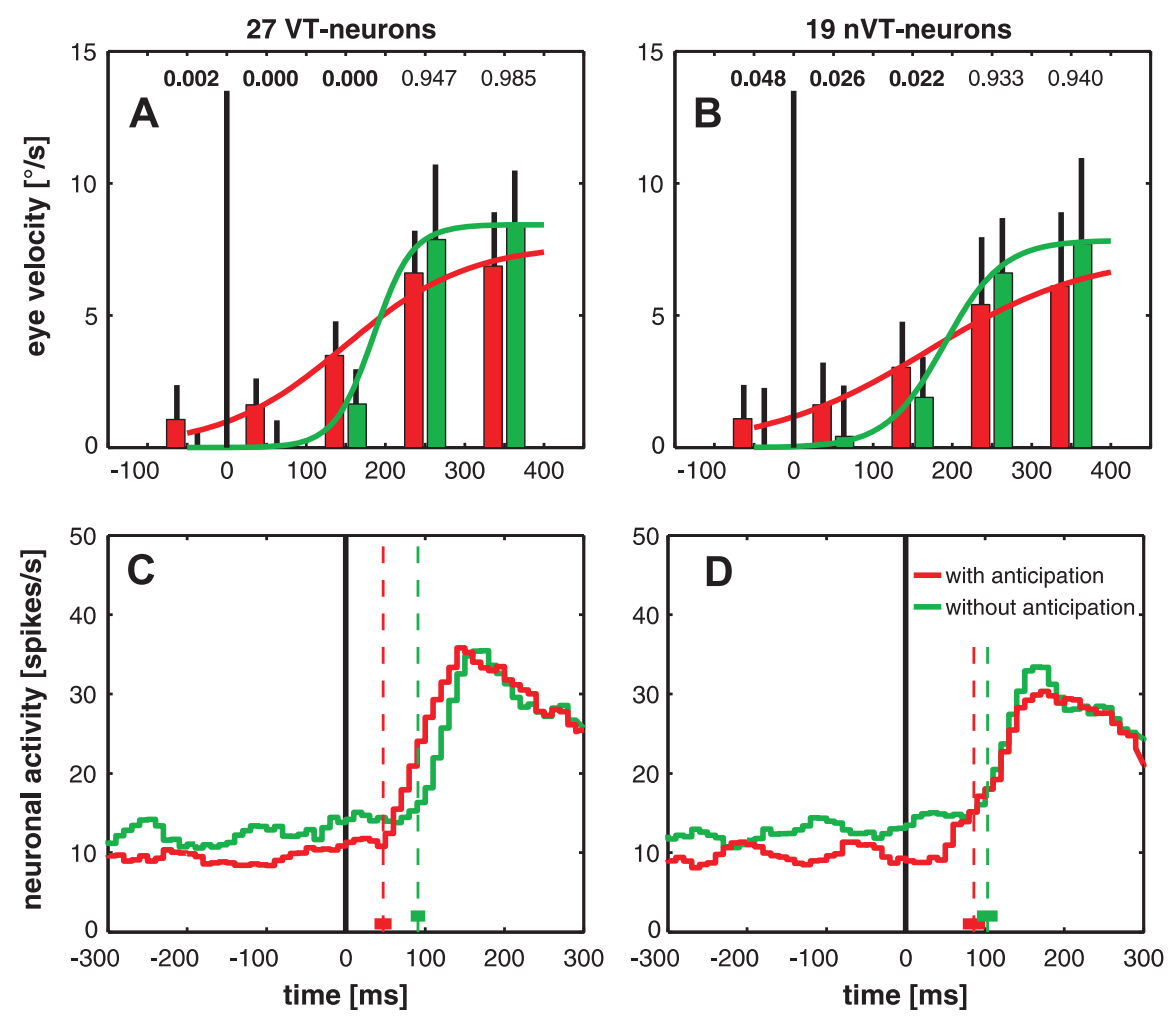

Fig. 2. Eye movements and population responses during anticipatory pursuit eye movements. Data related to the anticipation condition are drawn in red; data related to the control condition without anticipation are drawn in green. Time zero indicates the appearance of the moving target. On the left, the results obtained from 27 VT neurons with extra-retinal response properties are presented. On the right, the results from 19 nVT neurons without extra-retinal responses are shown. Eye velocity is computed in 100 ms time bins ( $\mathrm{A}$ and $\mathrm{B}$ ), the population responses in $10 \mathrm{~ms}$ bins ( $\mathrm{C}$ and $\mathrm{D})$. Direction of target movement was in the preferred direction of each recorded neuron. The latency of every neuron was determined independently, the vertical dashed lines represent the mean values, and the related horizontal bars show the standard errors. Note that the latency of the VT neurons was significantly shorter in the prediction condition shown in C, this difference was absent for the nVT neurons shown in extra-retinal (modified from (Ilg, 2003)). (For interpretation of the references to colour in this figure legend, the reader is referred to the web version of this article.)
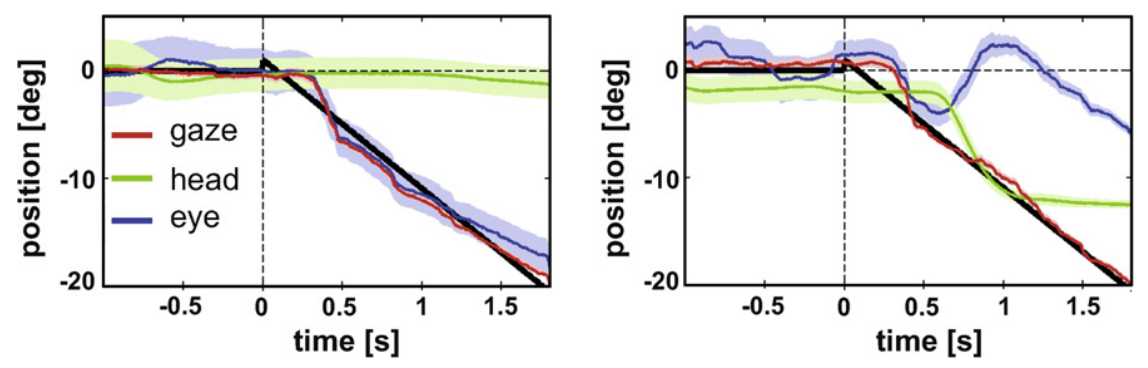

Fig. 3. Two different tracking conditions. In the left diagram, the monkey was instructed to track the target by an isolated eye movement only. In the right, the monkey was asked to perform a combined eye and head movement. The mean gaze, head, and eye position are presented based on 10 trials each together with the resulting standard error. Target position is indicated by the black profile. Time zero represents the onset of target movement. Note that the resulting gaze movements are very similar in both conditions. The reason for the standard error of the head position in the eye movement only condition was the variability of head position from trial to trial (modified from (Ilg et al., 2004)).

prediction condition lies in the extra-retinal response properties. In this condition, the monkeys performed smooth pursuit before the target appeared. The nVT neurons, whose activity is not influenced by the eye movements, only responded to visual motion signals and did therefore not have different latencies (Ilg, 2003). Clearly, these results support expectation put forward previously that the activity of VT neurons in MSTl is influenced by the ongoing eye movements.

\section{Origin of extra-retinal response properties}

In summary, there are two lines of evidence for the existence of eye movement-related signals in the activity of VT neurons. But what is the origin of the extra-retinal properties? The extra-retinal responses related to the ongoing eye movements might originate from the proprioceptors of the extra-ocular muscles or, alternatively, might represent the copy of the efferent command. These two possibilities parallel the general debate about the origin of information related to the actual eye position. According to the inflow theory, the Sherringtonian point of view, receptors in the extra-ocular muscles send this information to the central nervous system. In contrast, the outflow theory, the Helmholtzian view, states that eye position information arises from copies of the motor program (for review see (Grusser, 1986)).

The proprioceptive origin of the eye-movement-related signals can be definitively ruled out for two reasons: first, the density as well as the types of proprioceptive elements in the extra-ocular muscles of rhesus monkeys are unknown at present, the twitch 


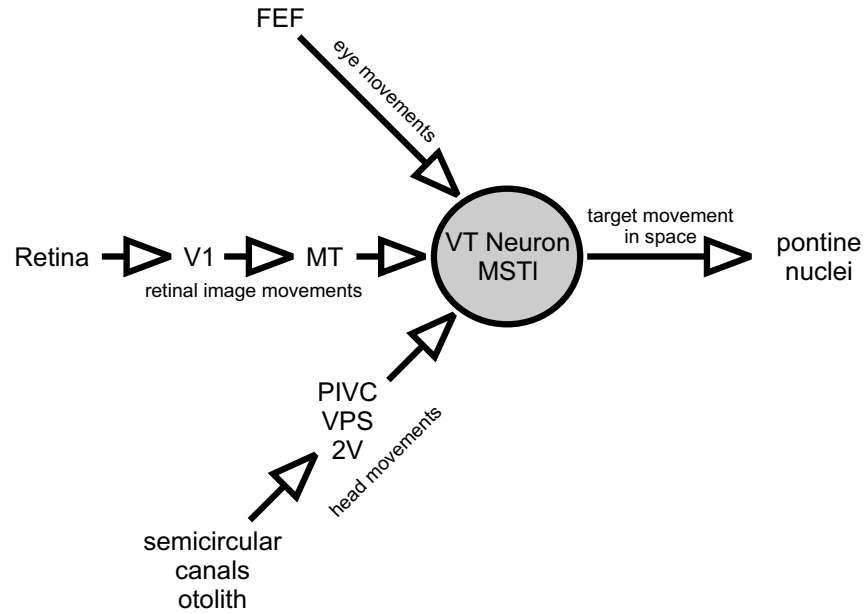

Fig. 4. Convergence of different inputs on visual tracking (VT) neurons recorded from MSTl. VT neurons convey information related to target movement in space to the pontine nuclei, which provide the input to the cerebellum and extra-ocular motor neurons. See text for further explanations.

reflex in the extra-ocular muscles is absent (Keller \& Robinson, 1971). Second, as addressed above, the average response latency of VT neurons is shorter than the eye-movement latency.

Alternatively, in accordance with the outflow theory, the extraretinal response properties might arise as an efference copy from the motor-neurons innervating the extra-ocular muscles. In order to test this hypothesis, we trained monkeys to track a moving target either by an isolated eye-movement or be a combined eye and head movements. The color of the moving target indicated which tracking behavior had to be executed: a red target indicated isolated eye movements; a green target signaled combined eye and head movement. The gaze movements in both tracking conditions were very similar (see Fig. 3). However, the eye-in-head movements (and therefore the activity of the motor-neurons) were entirely different (Ilg, Schumann, \& Thier, 2004).

We recorded the pursuit responses of VT neurons from MSTl in these two tracking conditions. Our statistical analysis revealed a significant difference between responses during pursuit in preferred and non-preferred direction, but no significant difference between the different tracking conditions. Therefore, we can clearly dismiss the possibility that the eye-movement-related activity is an efference copy in the strict sense, i.e. arises from the motor-neurons in the brainstem. To further characterize the nature of the pursuit-related activity, we performed experiments in which we combined a pursuit task with vestibular stimulation. While the monkeys were sinusoidally rotated, they had to track a target moving at a different frequency. The spectral analysis of the obtained responses showed that the neurons responded to the frequency of gaze and target movement, but not to the frequency of vestibular stimulation. Finally, a correlation analysis revealed that the activity is most closely related to target motion in space (Ilg et al., 2004). However, this does not answer the question as to the origin of the eye-movement-related signal. Our present hypothesis that this signal originates from the frontal eye field is currently under investigation (Fig. 4).

The last question addresses the origin of the head movementrelated signals. Since these signals are observed during passive vestibular stimulation, the only possible sources are the semicircular channels of the inner ear. It was proposed earlier that area MST might be included in the processing of vestibular information, i.e. that area MST receives information from the vestibular cortex such as the parietal insular vestibular cortex (PIVC), the visual posterior sylvian cortex (VPS) or area 2v (Guldin \& Grusser, 1998). In the meantime, sensitivity to vestibular stimulation was reported from the ventral intra-parietal area VIP (Klam \& Graf, 2006; Schlack, Hoffmann, \& Bremmer, 2002). These various cortical areas receive their vestibular information from the semicircular canals and the otolith via a relay station in the thalamus (Meng, May, Dickman, \& Angelaki, 2007).

\section{Contributions of MST to goal-directed behavior}

So far, the neuronal activity observed in MSTl was always discussed in terms of smooth pursuit eye movements. However, what is the overall role of MSTI? Is this area a processor of retinal image motion, eye and head movement-related signals whose only function is the generation of this type of eye movements? Or are there other functions carried into execution by MSTl? In order to answer this question, I will first focus on goal-directed behavior in general and subsequently address motion perception in the absence of any behavior.

Once we had identified the location of MSTl by our single-unit recordings, we addressed the consequences of an artificial increase or decrease of neuronal activity in this area. To increase the activity, we applied intra-cortical micro-stimulation through the same micro-electrodes which were used for recordings. To decrease the activity, we exchanged the micro-electrode with a glass pipette and injected small amounts of the GABA agonist muscimol. In short, we were able to replicate earlier findings regarding the execution of smooth pursuit eye movements (Ilg \& Schumann, 2007): We observed an acceleration of eye speed caused by the microstimulation $(200 \mathrm{~Hz}$ of bipolar pulses, pulse length $200 \mu \mathrm{s}, 50$ to $80 \mu \mathrm{A}, 200 \mathrm{~ms}$ delayed with respect to the onset of pursuit target) if pursuit was performed in the preferred direction of the recording site (Born, Groh, Zhao, \& Lukasewycz, 2000; Komatsu \& Wurtz, 1989). For this analysis, we determined the post-saccadic eye velocity within a $50 \mathrm{~ms}$ time window. Interestingly, the effect of micro-stimulation was significantly larger if the pursuit target was switched off during stimulation. Micro-stimulation during fixation of a stationary target did not result in an eye movement. Following the injection of muscimol $(<4 \mu \mathrm{l}$, concentration $5 \mathrm{mg} / \mathrm{ml}$ in $\mathrm{H}_{2} \mathrm{O}$ ), we observed a reduction of steady-state gain for ipsiversive target movement only; pursuit towards a contraversively moving target was not affected (Dursteler \& Wurtz, 1988; Dursteler, Wurtz, \& Newsome, 1987; Yamasaki \& Wurtz, 1991). In addition to the execution of eye movements, we trained our monkeys to perform hand movements towards moving visual targets. Following the GO signal indicated by a color change from red to blue of the fixation target, the monkeys had to reach out towards the moving target which appeared 200 to $500 \mathrm{~ms}$ prior to the GO signal. Pointing was measured using a touch screen in front of the projection screen. Since we switched off the target as soon as the hand movement started, the pointing of the monkeys was ballistic.

During micro-stimulation which started simultaneously with the GO signal, we observed a shift of the pointing movement into the direction of the preferred direction of the stimulation site in 28 out of 29 stimulation sites. In most cases, the preferred direction of the stimulation site was identical to the direction of the stimulation effect on eye and hand movement. In case of the transient lesion caused by the muscimol injection, we observed a shift in pointing contraversive to the injection site, independent of the direction of target motion (Ilg \& Schumann, 2007).

In order to explain the effects of artificial increase and decrease of activity, it is important to consider the concept of cortical columns (Mountcastle, 1957) which is most likely present in MST (Britten, 1998). Only if the tip of the electrode is centered within a cortical column, whose size was estimated to $0.5 \mathrm{~mm}$ (Britten, 1998), the output of this particular column is augmented and the 
effect of micro-stimulation is evident. It was estimated earlier that the spread of activity given by our stimulation parameters is in the order of $0.1 \mathrm{~mm}$ (Stoney, Thompson, \& Asanuma, 1968). If the electrode is close to the border between two columns, the stimulation effect is balanced. In 93 out of 136 stimulation sites we obtained significant effects on eye movements, most likely these sites were all centered within a cortical column. The fact that we observed a larger effect in the temporary absence of the pursuit target is another argument for the processing of retinal and extra-retinal signals in MST. In the presence of the target, the artificial activity is combined with retinal image motion and eye-movement-related signals. In the absence of the target, the artificial activity is combined with eye-movement-related activity only.

In contrast, the spread of muscimol was estimated in the order of $3 \mathrm{~mm}$ (Martin, 1991), which means that the transient lesions affected a much larger area. The reason for the observed ipsiversive pursuit deficit following a unilateral MST lesion is still a matter of debate. It seems less likely that an over-representation of neurons with ipsiversive preferred direction is able to explain this deficit. More convincing is an explanation based on functional grouping of the cortical output: neurons with ipsiversive preferred directions constitute the cortical output to subcortical centers as the pretectum (Ilg \& Hoffmann, 1993) or the pontine nuclei. On the other hand, neurons with contraversive preferred directions project to the contralateral hemisphere (Hoffmann, Distler, \& Ilg, 1992). So a unilateral lesion affects only ipsiversive pursuit. There was a striking difference between the deficits observed in eye and hand movements, respectively. The ipsiversive pursuit deficit indicates parallel processing of both hemispheres. In contrast, hand movements seem to depend on the balanced output of both hemispheres. Thus, the contraversive deviation of pointing can be explained by the missing ipsiversive component reflecting the inactivated hemisphere.

These results concerning the precision of hand movements clearly demonstrate that MSTl is not only important for generating smooth pursuit eye movements. So MSTl is no specific preprocessor of visual motion for smooth pursuit. But is MSTl also involved in the processing if no goal-directed behavior at all is prepared if the monkey is engaged in a psychophysical motion task? Do MSTl neurons only respond to retinal image motion, eye and head movement as described earlier during the execution of goal-directed behavior or is there a general coding of inferred motion as proposed by others (Assad \& Maunsell, 1995)? It is important to note here that after appropriate training, neurons recorded from MT respond even to an arrow representing a moving stimulus (Schlack \& Albright, 2007).

\section{Contributions of MT and MST to motion perception}

In order to address visual motion processing in areas MT and MST underlying motion perception we initially decided to use second-order motion stimuli and moving visual and auditory stimuli (Ilg \& Churan, 2004). We successfully trained our monkeys to report the direction of a moving stimulus in a direction discrimination task (DDT). During fixation of a stationary target, the actual motion stimulus was displayed centered on the receptive field of the recorded neuron. Subsequently, two saccade targets appeared and the monkey made a saccade to the target that was in the direction of the movement.

We used motion stimuli defined by fourier-motion (coherent motion), drift-balanced and theta motion stimuli. Each stimulus consisted of a rectangle adjusted to the size of the receptive field of the recorded neuron. In the case of fourier-motion, this moving rectangle comprised a constant pattern of coherently moving dots. For the drift-balanced motion, dots within the moving rectangle changed their luminance. Drift-balanced motion can be envisaged as a moving window in a structured proximal plane through which a second, distal plane can be seen. Finally, in the case of theta motion, the rectangle consisted of dots moving to the left while the rectangle moved to the right or vice versa. We have shown earlier that human subjects are able to track these second-order motion stimuli (Butzer, Ilg, \& Zanker, 1997), despite the initiation of pursuit being mainly dominated by the Fourier component (Lindner \& Ilg, 2000).

The moving visual and auditory stimuli were generated by a one-dimensional array of LEDs and loud-speakers which were sequentially activated (Ilg \& Churan, 2004). We applied visual, auditory or bimodal moving stimuli. To our surprise, we did not find a general response to moving stimuli in MT and MST despite the fact that our monkeys performed all experiments close to $100 \%$ correctly. In the case of fourier-motion and visual motion, we observed directionally selective responses in 38 MT and 68 MST neurons, as expected. For the drift-balanced stimulus, about half of our recorded MST neurons gave significant response as also reported by others (Albright, 1992; O'Keefe and Movshon, 1998). When we applied the theta motion stimulus, all recorded neurons responded to the motion of the individual dots, not of the entire object. It seemed as if the neurons inverted their preferred direction. Using a slightly different stimulus, Anthony Movshon and colleagues arrived at the same conclusion, namely that MT neurons represent local not global motion signals (Majaj, Carandini, \& Movshon, 2007).

Finally, we recorded from 6 MT and 27 MST neurons that showed significant responses to the moving visual and bimodal stimulus, but no response at all was observed in these neurons during presentation of the moving sound source.

In contrast to our own results, the importance of MT and MST for motion perception has been convincingly shown in single-unit studies (Bisley \& Pasternak, 2000; Britten, Newsome, Shadlen, \& Celebrini, 1996; Celebrini \& Newsome, 1994), in lesions studies (Newsome \& Pare, 1988; Rudolph \& Pasternak, 1999) and in micro-stimulation studies (Bisley, Zaksas, \& Pasternak, 2001; Celebrini \& Newsome, 1995; Ditterich, Mazurek, \& Shadlen, 2003). However, the common denominator of all these studies, clearly differing from our own study, is that there was a visual stimulus present which triggered the perception. There is only a single study demonstrating that neurons in this part of the brain respond to inferred motion in the absence of any physical stimulus (Assad \& Maunsell, 1995).

In contrast to the experiments using second-order motion and moving sound sources, we decided to analyze the effect of the temporal background properties on perception and neuronal responses. The detrimental effect of background flicker on motion perception and on the sharpness of directional tuning of 155 neurons recorded from MT and MST argues for the contribution of these areas to motion perception (Churan \& Ilg, 2002). In a series of psychophysical experiments, we applied the direction discrimination task (DDT) described above and determined the thresholds of stimulus presentation for human subjects and monkeys. If the fourier-motion stimulus was presented in front of a dynamic random dot pattern (flicker), the stimulus presentation threshold was elevated, in humans from $25 \mathrm{~ms}$ to $60 \mathrm{~ms}$, in monkeys from $99 \mathrm{~ms}$ to $280 \mathrm{~ms}$. In addition, we determined the sharpness of the directionally selective responses expressed as d' for fourier-motion presentation in front of a static and flickering background. For the flickering background, d' of the neuronal responses was reduced approximately $30 \%$ compared to d' obtained in the static background condition. The comparable effects of background flicker on motion perception and neuronal responses recorded from MT and MST suggest the notion that MT and MST are indeed involved in the processing underlying motion perception as suggested by 
many other studies. But there is also clear evidence that MT and MST are definitively not the final stages with respect to perception of motion.

\section{Acknowledgments}

I thank all my students for their enthusiasm for scientific questions and their solution. In particular, I am grateful to Inés Trigo-Damas for the single-unit recordings from MT presented here, Simon Jakob for critical proofreading, Ute Grosshennig for successfully handling the frequently occurring problems in the lab, and Peter Thier for continuous support over many years. Financial support from the German Research Council (DFG IL 34/6-1) made these studies possible.

\section{References}

Albright, T. D. (1992). Form-cue invariant motion processing in primate visual cortex. Science, 255, 1141-1143.

Allman, J. M., \& Kaas, J. H. (1971). A representation of the visual field in the cauda third of the middle temporal gyrus of the owl monkey (Aotus trivirgatus). Brain Research, 31(1), 85-105.

Assad, J. A., \& Maunsell, J. H. R. (1995). Neuronal correlates of inferred motion in primate posterior parietal cortex. Nature, 373, 518-521.

Barnes, G. R., \& Asselman, P. T. (1991). The mechanism of prediction in human smooth pursuit eye movements. Journal of Physiology, 439, 439-461.

Bisley, J. W., \& Pasternak, T. (2000). The multiple roles of visual cortical areas MT/ MST in remembering the direction of visual motion. Cerebral Cortex, 10 1053-1065.

Bisley, J. W., Zaksas, D., \& Pasternak, T. (2001). Microstimulation of cortical area MT affects performance on a visual working memory task. Journal of Neurophysiology, 85(1), 187-196.

Born, R. T., Groh, J. M., Zhao, R., \& Lukasewycz, S. J. (2000). Segregation of object and background motion in visual area MT: Effects of microstimulation on eye movements. Neuron, 26(3), 725-734

Bradley, D. C., Maxwell, M., Andersen, R. A., Banks, M. S., \& Shenoy, K. V. (1996) Mechanisms of heading perception in primate visual cortex. Science, 273 1544-1547.

Britten, K. H. (1998). Clustering of response selectivity in the medial superio temporal area of extrastriate cortex in the macaque monkey. Visual Neuroscience, 15(3), 553-558.

Britten, K. H., Newsome, W. T., Shadlen, M. N., \& Celebrini, S. (1996). A relationship between behavioral choice and the visual responses of neurons in macaque MT. Visual Neuroscience, 13, 87-100.

Butzer, F., Ilg, U. J., \& Zanker, J. M. (1997). Smooth-pursuit eye movements elicited by first-order and second-order motion. Experimental Brain Research, 115(1), $61-70$.

Celebrini, S., \& Newsome, W. T. (1994). Neuronal and psychophysical sensitivity to motion signals in extrastriate area MST of the macaque monkey. Journal of Neuroscience, 14(7), 4109-4124.

Celebrini, S., \& Newsome, W. T. (1995). Microstimulation of extrastriate area MST influences performance on a direction discrimination task. Journal of Neurophysiology, 73, 437-448.

Churan, J., \& Ilg, U. J. (2002). Flicker in the visual background impairs the ability to process a moving visual stimulus. The European Journal of Neuroscience, 16(6), $1151-1162$

Desimone, R., \& Ungerleider, L. G. (1986). Multiple visual areas in the caudal superior temporal sulcus of the Macaque. The Journal of Comparative Neurology, $248,164-189$

Dicke, P. W., \& Thier, P. (1999). The role of cortical area MST in a model of combined smooth eye-head pursuit. Biological Cybernetics, 80, 71-84.

Ditterich, J., Mazurek, M. E., \& Shadlen, M. N. (2003). Microstimulation of visual cortex affects the speed of perceptual decisions. Nature Neuroscience, 6(8) 891-898.

Dubner, R., \& Zeki, S. M. (1971). Response properties and receptive fields of cells in an anatomically defined region of the superior temporal sulcus in the monkey. Brain Research, 35(2), 528-532.

Duffy, C. J., \& Wurtz, R. H. (1991). Sensitivity of MST neurons to optic flow stimuli. I. A continuum of response selectivity to large-field stimuli. Journal of Neurophysiology, 65(6), 1329-1345.

Dursteler, M. R., \& Wurtz, R. H. (1988). Pursuit and optokinetic deficits following chemical lesions of cortical areas MT and MST. Journal of Neurophysiology, 60(3) 940-965.

Dursteler, M. R., Wurtz, R. H., \& Newsome, W. T. (1987). Directional pursuit deficits following lesions of the foveal representation within the superio temporal sulcus of the macaque monkey. Journal of Neurophysiology, 57(5), 1262-1287.

Freyberg, S., \& Ilg, U. J. (2007). Anticipatory smooth-pursuit eye movements in man and monkey. Experimental Brain Research.

Grusser, O. J. (1986). Interaction of efferent and afferent signals in visual perception. A history of ideas and experimental paradigms. Acta Psychologica, 63(1-3), 3-21.
Guldin, W. O., \& Grusser, O. J. (1998). Is there a vestibular cortex? Trends in Neurosciences, 21(6), 254-259.

Hawken, M. J., Parker, A. J., \& Lund, J. S. (1988). Laminar organization and contrast sensitivity of direction-selective cells in the striate cortex of the Old World monkey. Journal of Neuroscience, 8(10), 3541-3548.

Hoffmann, K. P., Distler, C., \& Ilg, U. (1992). Callosal and superior temporal sulcus contributions to receptive field properties in the macaque monkey's nucleus of the optic tract and dorsal terminal nucleus of the accessory optic tract. The Journal of Comparative Neurology, 321(1), 150-162.

Hubel, D. H., \& Wiesel, T. N. (1968). Receptive fields and functional architecture of monkey striate cortex. Journal of Physiology, 195, 215-243.

Ilg, U. J. (2003). Visual-tracking neurons in area MST are activated during anticipatory pursuit eye movements. Neuroreport, 14(17), 2219-2223.

Ilg, U. J., \& Churan, J. (2004). Motion perception without explicit activity in areas MT and MST. Journal of Neurophysiology, 92(3), 1512-1523.

Ilg, U. J., \& Hoffmann, K. P. (1993). Functional grouping of the cortico-pretectal projection. Journal of Neurophysiology, 70(2), 867-869.

Ilg, U. J., \& Schumann, S. (2007). Primate area MST-1 is involved in the generation of goal-directed eye and hand movements. Journal of Neurophysiology, 97(1), 761-771.

Ilg, U. J., Schumann, S., \& Thier, P. (2004). Posterior parietal cortex neurons encode target motion in world-centered coordinates. Neuron, 43(1), 145-151.

Ilg, U. J., \& Thier, P. (1999). Eye movements of rhesus monkeys directed towards imaginary targets. Vision Research, 39(12), 2143-2150.

Ilg, U. J., \& Thier, P. (2003). Visual tracking neurons in primate area MST are activated by smooth-pursuit eye movements of an "imaginary" target. Journal of Neurophysiology, 90(3), 1489-1502.

Kawano, K., Sasaki, M., \& Yamashita, M. (1984). Response properties of neurons in posterior parietal cortex of monkey during visual vestibular stimulation I visual tracking neurons. Journal of Neurophysiology, 51(2), 340-351.

Keller, E. L., \& Robinson, D. A. (1971). Absence of a stretch reflex in extraocular muscles of the monkey. Journal of Neurophysiology, 34(5), 908-919.

Klam, F., \& Graf, W. (2006). Discrimination between active and passive head movements by macaque ventral and medial intraparietal cortex neurons. Journal of Physiology, 574(Pt 2), 367-386.

Komatsu, H., \& Wurtz, R. H. (1989). Modulation of pursuit eye movements by stimulation of cortical areas MT and MST. Journal of Neurophysiology, 62(1), 31-47.

Kowler, E., \& Steinman, R. M. (1979). The effect of expectations on slow oculomotor control. I. Periodic target steps. Vision Research, 19(6), 619-632.

Lanman, J., Bizzi, E., \& Allum, J. (1978). The coordination of eye and head movement during smooth pursuit. Brain Research, 153, 39-53.

Lindner, A., \& Ilg, U. J. (2000). Initiation of smooth-pursuit eye movements to firstorder and second-order motion stimuli. Experimental Brain Research, 133(4), 450-456.

Majaj, N. J., Carandini, M., \& Movshon, J. A. (2007). Motion integration by neurons in macaque MT is local, not global. Journal of Neuroscience, 27(2), 366-370.

Martin, J. H. (1991). Autoradiographic estimation of the extend of reversible inactivation produced by microinjection of lidocaine and muscimol in the rat. Neuroscience Letters, 127, 160-164.

Maunsell, J. H., \& van Essen, D. C. (1983). The connections of the middle tempora visual area (MT) and their relationship to a cortical hierarchy in the macaque monkey. Journal of Neuroscience, 3(12), 2563-2586.

Meng, H., May, P. J., Dickman, J. D., \& Angelaki, D. E. (2007). Vestibular signals in primate thalamus: Properties and origins. Journal of Neuroscience, 27(50), $13590-13602$

Mikami, A., Newsome, W. T., \& Wurtz, R. H. (1986). Motion selectivity in macaque visual cortex. II. Spatiotemporal range of directional interactions in MT and V1. Journal of Neurophysiology, 55(6), 1328-1339.

Mountcastle, V. B. (1957). Modality and topographic properties of single neurons of cat's somatic sensory cortex. Journal of Neurophysiology, 20(4), 408-434.

Movshon, J. A., \& Newsome, W. T. (1996). Visual response properties of striate cortical neurons projecting to area MT in macaque monkeys. Journal of Neuroscience, 16(23), 7733-7741.

Nassi, J. J., Lyon, D. C., \& Callaway, E. M. (2006). The parvocellular LGN provides a robust disynaptic input to the visual motion area MT. Neuron, 50(2), 319-327.

Newsome, W. T \& Pare, E B (1988). A selective impairment of motion perception following lesions of the middle temporal visual area MT. Journal of Neuroscience.

Newsome, W. T., Wurtz, R. H., \& Komatsu, H. (1988). Relation of cortical areas MT and MST to pursuit eye movements. II. Differentiation of retinal from extraretinal inputs. Journal of Neurophysiology, 60(2), 604-620.

O'Keefe, L. P., \& Movshon, J. A. (1998). Processing of first- and second-order motion signals by neurons in area MT of the macaque monkey. Visual Neuroscience, 15 305-317.

Ponce, C. R., Lomber, S. G., \& Born, R. T. (2008). Integrating motion and depth via parallel pathways. Nature Neuroscience.

Rudolph, K., \& Pasternak, T. (1999). Transient and permanent deficits in motion perception after lesions of cortical areas MT and MST in the macaque monkey. Cerebral Cortex, 9(1), 90-100.

Schlack, A., \& Albright, T. D. (2007). Remembering visual motion: neural correlates of sssociative plasticity and motion recall in cortical area MT. Neuron, 53(6), 881-890.

Schlack, A., Hoffmann, K. P., \& Bremmer, F. (2002). Interaction of linear vestibular and visual stimulation in the macaque ventral intraparietal area (VIP). The European Journal of Neuroscience, 16(10), 1877-1886. 
Sincich, L. C., Park, K. F., Wohlgemuth, M. J., \& Horton, J. C. (2004). Bypassing V1: A direct geniculate input to area MT. Nature Neuroscience, 7(10), 1123-1128.

Stoney, S. D., Jr., Thompson, W. D., \& Asanuma, H. (1968). Excitation of pyramidal tract cells by intracortical microstimulation: Effective extent of stimulating current. Journal of Neurophysiology, 31(5), 659-669.

Tanaka, K., Hikosaka, K., Saito, H., Yukie, M., Fukada, Y., \& Iwai, E. (1986) Analysis of local and wide-field movements in the superior temporal visual areas of the macaque monkey. Journal of Neuroscience, 6(1), 134-144.
Thier, P., \& Erickson, R. G. (1992). Responses of visual-tracking neurons from cortical area MST-I to visual, eye and head motion. The European Journal of Neuroscience, 4(6), 539-553.

Treue, S., \& Maunsell, J. H. R. (1996). Attentional modulation of visual motion processing in cortical areas MT and MST. Nature, 382(8), 539-541.

Ungerleider, L. G., \& Desimone, R. (1986). Cortical connections of visual area MT in the macaque. The Journal of Comparative Neurology, 248(2), 190-222.

Yamasaki, D. S., \& Wurtz, R. H. (1991). Recovery of function after lesions in the superior temporal sulcus in the monkey. Journal of Neurophysiology, 66, 651-673. 